\title{
Combination of soya pulp and Bacillus coagulans lilac-01 improves intestinal bile acid metabolism without impairing the effects of prebiotics in rats fed a cholic acid-supplemented diet
}

\author{
Yeonmi Lee ${ }^{1}$, Reika Yoshitsugu ${ }^{1}$, Keidai Kikuchi ${ }^{1}$, Ga-Hyun Joe ${ }^{1}$, Misaki Tsuji ${ }^{1}$, Takuma Nose ${ }^{1}$, \\ Hidehisa Shimizu $^{1,2}$, Hiroshi Hara ${ }^{1}$, Kimiko Minamida ${ }^{3}$, Kazunori Miwa ${ }^{3}$ and Satoshi Ishizuka ${ }^{1}{ }^{\prime}$ \\ ${ }^{1}$ Research Faculty of Agriculture, Hokkaido University, Sapporo 060-8589, Japan \\ ${ }^{2}$ Faculty of Life and Environmental Science, Shimane University, Matsue 690-8504, Japan \\ ${ }^{3}$ Arterio Bio Co., Ltd, Otaru 047-0261, Japan \\ (Submitted 14 March 2016 - Final revision received 28 April 2016 - Accepted 9 May 2016 - First published online 13 June 2016)
}

\section{Abstract}

Intestinal bacteria are involved in bile acid (BA) deconjugation and/or dehydroxylation and are responsible for the production of secondary BA. However, an increase in the production of secondary BA modulates the intestinal microbiota due to the bactericidal effects and promotes cancer risk in the liver and colon. The ingestion of Bacillus coagulans improves constipation via the activation of bowel movement to promote defaecation in humans, which may alter BA metabolism in the intestinal contents. BA secretion is promoted with high-fat diet consumption, and the ratio of cholic acid (CA):chenodeoxycholic acid in primary BA increases with ageing. The dietary supplementation of CA mimics the BA environment in diet-induced obesity and ageing. We investigated whether B. coagulans lilac-01 and soya pulp influence both BA metabolism and the maintenance of host health in CA-supplemented diet-fed rats. In CA-fed rats, soya pulp significantly increased the production of secondary BA such as deoxycholic acid and $\omega$-muricholic acids, and soya pulp ingestion alleviated problems related to plasma adiponectin and gut permeability in rats fed the CA diet. The combination of B. coagulans and soya pulp successfully suppressed the increased production of secondary BA in CA-fed rats compared with soya pulp itself, without impairing the beneficial effects of soya pulp ingestion. In conclusion, it is possible that a combination of prebiotics and probiotics can be used to avoid an unnecessary increase in the production of secondary BA in the large intestine without impairing the beneficial functions of prebiotics.

\section{Key words: Bile acids: Soya pulp: Bacillus coagulans lilac-01: Synbiotics: Gut permeability}

Prebiotics are non-digestible dietary factors that provide a beneficial physiological effect for the host by selectively stimulating the growth or activity of a limited number of indigenous bacteria. A combination of prebiotics and probiotics known as synbiotics, as originally proposed by Gibson \& Roberfroid $^{(1)}$, shows a synergistic influence on obesity, diabetes, fatty liver and colitis ${ }^{(2)}$. We have also demonstrated a synergistic effect of synbiotcs in combination with Bifidobacterium breve and raffinose on the intestinal epithelial proliferation in rats ${ }^{(3)}$. Orally introduced bacteria usually suffer because of the gastric acid, digestive enzymes and bile acids (BA) secreted from the gastrointestinal tissues. Prebiotics promote their viability and activity in the luminal environment of the gastrointestinal tract. Bacillus coagulans is one of the spore-forming lactic acid bacteria that tolerate heat, oxygen, acid and $\mathrm{BA}^{(4)}$. Soya pulp, which was supplemented to the rats in this study, is a by-product after extraction of soya milk. Some types of oligosaccharides observed in soyabean can be included in the soya pulp as well ${ }^{(5)}$, indicating that soya pulp has potential as a prebiotic. B. coagulans lilac-01 was previously isolated and the synbiotics of the strain with soya pulp as a prebiotic are effective for improving bowel movement and faecal properties in functionally constipated patients in a double-blinded and randomised study ${ }^{(6)}$.

BA, which are steroids synthesised from cholesterol, are made in the liver as primary BA and are secreted into the luminal environment of the intestine to help digestion and absorption of dietary lipids and hydrophobic nutrients ${ }^{(7)}$. Most of the BA are reabsorbed in the ileum and return to the liver through the blood stream in a process known as enterohepatic circulation $^{(8,9)}$. Secondary BA are produced by some intestinal bacteria via the deconjugation and dehydroxylation of primary $\mathrm{BA}$. There is a mutual relationship between the intestinal BA metabolism and the intestinal bacterial composition. Some bacteria influence BA composition via deconjugation, dehydorgenation, epimerisation and dehydroxylation ${ }^{(10)}$. Both aerobic and anaerobic bacteria, such as Lactobacillus ${ }^{(11-13)}$,

Abbreviations: ALT, alanine aminotransaminase; AST, aspartate aminotransferase; BA, bile acid; CA, cholic acid; CDCA, chenodeoxycholic acid; DCA, deoxycholic acid; LCA, lithocholic acid; Syn, synbiotic diet.

* Corresponding author: S. Ishizuka, fax +81 11706 2811, email zuka@chem.agr.hokudai.ac.jp 
Bifidobacterium $^{(14,15)}$ and Enterococcus ${ }^{(16)}$, possess deconjugation activity. Dehydroxylation activity is detected in Clostridium $^{(17)}$. Increased secondary BA can cause colorectal and hepatic carcinogenesis via the induction of necrosis with inflammation and DNA damage ${ }^{(18)}$.

The consumption of a high-fat diet increases BA secretion, especially that of the secondary BA such as deoxycholic acid (DCA) and lithocholic acid (LCA $)^{(19)}$. Ageing raises the cholic acid (CA):chenodeoxycholic acid (CDCA) ratio of the BA composition in rodents ${ }^{(20)}$. In humans, BA secreted from the liver are unchanged by age ${ }^{(21)}$. However, there is an increase in the serum DCA concentration in response to meal consumption, suggesting an increase in the production of secondary BA in the intestine. We used dietary CA supplementation at a moderate level in rats to mimic the increase in both BA secretion and the CA:CDCA ratio by ageing with a high-fat diet. As a result, in the CA-fed rats, an increase in the relative abundance of the phylum Firmicutes and a decrease in the phylum Bacteroidetes were observed $^{(22)}$. Such alterations of the gut bacterial composition are similar to that observed in obese individuals or with high-fat diet consumption. The results indicate that CA supplementation at a moderate level in the diet is involved in some aspects of metabolic disorders. In addition, probiotics, prebiotics and synbiotics might influence the CA-induced dysregulations in host homoeostasis.

The aim of this study was to investigate whether the combination of B. coagulans with soya pulp influences BA excretion and host homoeostasis under CA-supplemented conditions.

\section{Methods}

\section{Animals}

This study was approved by the Hokkaido University Animal Committee. Animal care and use were in accordance with the Hokkaido University guidelines for the care and use of laboratory animals. After acclimation of the male Wistar rats (4 weeks old; Japan SLC, Inc.) with an AIN-93G $\operatorname{diet}^{(23)}$ for $7 d$, the rats were divided into five dietary groups and fed ad libitum a basal diet (B) or diets containing CA $(0.5 \mathrm{~g} / \mathrm{kg}$ diet $)$ in combination with B. coagulans lilac-01 at $10^{9}$ colony forming unit (CFU) $/ \mathrm{kg}$ diet and soya pulp for 8 weeks, as shown in Table 1 . The rats fed with the CA-supplemented diet were regarded as the control $(\mathrm{Ct})$ among the CA-fed groups. The probiotic diet (Pro) and the synbiotic diet (Syn) supplemented with CA contained either B. coagulans lilac-01 ( $10^{9} \mathrm{CFU} / \mathrm{kg}$ diet; Arterio Bio Co. Ltd) or a mixture of soya pulp and $B$. coagulans lilac-01 ( $10^{9} \mathrm{CFU} / \mathrm{kg}$ diet $)$, respectively. The prebiotic diet (Pre) supplemented with CA contained soya pulp as the fibre source. The rats were housed in an air-conditioned room at $22 \pm 2^{\circ} \mathrm{C}$ with $55 \pm 5 \%$ humidity, which was in the light cycle from 08.00 to 20.00 hours automatically.

After sampling the aorta and portal vein blood under anaesthesia with pentobarbital sodium salt $(50 \mathrm{mg} / \mathrm{kg}$ body weight, Somnopentyl; Kyoritsu Seiyaku Corporation), organs including the liver, kidneys, whole caecum, epididymal adipose tissue and retroperitoneal adipose tissue were collected. The caecal contents were diluted with ultra-pure water, and the $\mathrm{pH}$
Table 1. Diet compositions ( $\mathrm{g} / \mathrm{kg}$ diet)

\begin{tabular}{|c|c|c|c|c|c|}
\hline \multirow[b]{2}{*}{ Ingredients } & \multirow[b]{2}{*}{ B } & \multicolumn{4}{|c|}{$\mathrm{CA}$} \\
\hline & & $\mathrm{Ct}$ & Pro & Pre & Syn \\
\hline Maize starch* & 397 & 397 & 397 & 397 & 397 \\
\hline Casein† & 200 & 200 & 200 & 181.9 & 181.9 \\
\hline Dextrinł & 132 & 132 & 132 & 132 & 132 \\
\hline Sucrose§ & 100 & 100 & 100 & 96 & 96 \\
\hline Soyabean oil & 70 & 70 & 70 & $61 \cdot 7$ & $61 \cdot 7$ \\
\hline Crystalline cellulosell & 50 & 50 & 50 & 30.4 & $30 \cdot 4$ \\
\hline Mineral mixtureף & 35 & 35 & 35 & 35 & 35 \\
\hline Vitamin mixture ${ }^{\star \star}$ & 10 & 10 & 10 & 10 & 10 \\
\hline L-Cystine & 3 & 3 & 3 & 3 & 3 \\
\hline Choline bitartrate & 2.5 & 2.5 & $2 \cdot 5$ & 2.5 & $2 \cdot 5$ \\
\hline Cholic acid & - & 0.5 & 0.5 & 0.5 & 0.5 \\
\hline Soya pulp †† & - & - & - & 50 & - \\
\hline Bacillus coagulans lilac-01 & - & - & 5 & - & - \\
\hline Soya pulp with $B$. coagulans lilac-01 & - & - & - & - & 50 \\
\hline
\end{tabular}

CA, cholic acid; B, basal diet; Pro, probiotic diet; Pre, prebiotic; Syn, synbiotic diet. * Amylalpha; Chuo Shokuryou Co. Ltd.

† NZMP acid casein; Co-operative Group.

¥ TK16; Matsutani Chemical Industry Co., Ltd.

$\S$ Nippon Beet Sugar Manufacturing Co., Ltd.

II Ceolus PH-102; Asahi Kasei Chemicals Co.

II AIN-93 mineral mixture; MP Biomedicals, LLC.

** AIN-93 vitamin mixture; CLEA Japan Inc.

†† Kikkoman Soyfoods Co., Ltd.

was measured. The plasma and liver samples were stored at $-80^{\circ} \mathrm{C}$. The caecal contents and faeces were stored at $-40^{\circ} \mathrm{C}$ until the HPLC analysis.

\section{Gut permeability}

The rats were orally administered chromium (III) chloride hexahydrate (Wako Pure Chemical Industries, Ltd) solution with EDTA $2 \mathrm{Na}$ (Dojindo Laboratories) $2 \mathrm{~d}$ before being killed ${ }^{(24)}$. Urine samples were collected at $24 \mathrm{~h}$ after the oral administration, and urinary excretion of $\mathrm{Cr}$ was measured using an atomic absorption photometer (Z-5310; Hitachi High-Technologies Corporation).

\section{Liver and plasma parameters}

For aspartate aminotransferase (AST) and alanine aminotransaminase (ALT), aortic plasma was analysed using a transaminase CII test Wako kit (Wako Pure Chemical Industries, Ltd). NEFA were determined in aortic plasma using a NEFA C test Wako (Wako Pure Chemical Industries, Ltd). Adiponectin levels in the aortic plasma were measured using an adiponectin ELISA kit (Otsuka Pharmaceutical Co., Ltd). For lipid extraction, $100 \mathrm{mg}$ of liver was immersed in lipid-extraction solution (chloroform-methanol $=2: 1)^{(25)}$ and placed for $2 \mathrm{~d}$. The solvent in the extracts was corrected and evaporated spontaneously in the fume hood. The extracted lipids were dissolved with 2-propanol for measurement. TAG and cholesterol were determined using the TAG E-test and cholesterol E-test from Wako (Wako Pure Chemical Industries, Ltd), respectively.

\section{Bile acid analysis}

The BA were measured by UPLC/ESI-MS (Waters) with 23 -nor-5 $\beta$-cholanic acid-3 $\alpha, 12 \alpha$-diol (NDCA) as an internal standard according to our previous study ${ }^{(26)}$. The BA in the 
freeze-dried samples were extracted with ethanol and then purified with an HLB cartridge (Waters) according to the manufacturer's instruction. The BA in each sample were separated using an Acquity UPLC system equipped with a $\mathrm{BEH} \mathrm{C}_{18}$ column $(1.7 \mu \mathrm{M}$, $100 \times 2.0 \mathrm{~mm}$ i.d.) (Waters) and were analysed on a Quattro Premier XE quadrupole tandem MS (Waters). The BA measured in this study were as follows: $5 \beta$-cholanic acid- $3 \alpha, 7 \alpha, 12 \alpha$-triol (CA); $5 \beta$-cholanic acid- $3 \alpha, 6 \beta, 7 \alpha$-triol ( $\alpha$-muricholic acid; $\alpha \mathrm{MCA}$ ); $5 \beta$-cholanic acid- $3 \alpha, 6 \beta, 7 \beta$-triol ( $\beta$-muricholic acid; $\beta \mathrm{MCA}$ ); $5 \beta$ cholanic acid- $3 \alpha, 6 \alpha, 7 \beta$-triol ( $\omega$-muricholic acid; $\omega \mathrm{MCA}) ; 5 \beta$-cholanic acid- $3 \alpha, 6 \alpha$-diol (hyodeoxycholic acid; HDCA); $5 \beta$-cholanic acid$3 \alpha, 7 \beta$-diol; $5 \beta$-cholanic acid- $3 \alpha, 7 \alpha$-diol (CDCA); $5 \beta$-cholanic acid$3 \alpha, 12 \alpha$-diol (DCA); $5 \beta$-cholanic acid- $3 \alpha$-ol (LCA); $5 \beta$-cholanic acid- $3 \alpha, 6 \alpha, 7 \alpha$-triol; $5 \beta$-cholanic acid- $3 \alpha, 7 \beta, 12 \alpha$-triol (ursocholic acid; UCA); $5 \beta$-cholanic acid- $3 \alpha, 12 \alpha$-diol-7-one; $5 \beta$-cholanic acid-3 $\alpha$-ol-7-one; $5 \beta$-cholanic acid-3 $\alpha$-ol-12-one (12-oxolithocholic acid; 12oLCA); $5 \beta$-cholanic acid-12 $\alpha$-ol-3-one (3o12 $\alpha) ; \quad 5 \beta$-cholanic- $3 \alpha, 7 \alpha, 12 \alpha$-triol- $N$-(2-sulphoethyl)-amide (taurocholic acid; TCA); $5 \beta$-cholanic- $3 \alpha, 6 \beta, 7 \alpha$-triol- $N$ - $(2-$ sulphoethyl)-amide; $5 \beta$-cholanic- $3 \alpha, 6 \beta, 7 \beta$-triol- $N$-(2-sulphoethyl)amide; $5 \beta$-cholanic- $3 \alpha, 6 \alpha, 7 \beta$-triol- $N$-(2-sulphoethyl)-amide; $5 \beta$ cholanic- $3 \alpha, 6 \alpha$-diol- $N$-(2-sulphoethyl)-amide; $\quad 5 \beta$-cholanic- $3 \alpha$, $7 \alpha$-diol- $N$-(2-sulphoethyl)-amide; $\quad 5 \beta$-cholanic- $3 \alpha, 12 \alpha$-diol- $N$ - $(2-$ sulphoethyl)-amide; $\quad 5 \beta$-cholanic- $3 \alpha$-ol- $N$-(2-sulphoethyl)-amide; $5 \beta$-cholanic- $3 \alpha, 7 \alpha, 12 \alpha$-triol- $N$-(carboxymethl)-amide; $5 \beta$-cholanic$3 \alpha, 6 \alpha$-diol- $N$-(carboxymethl)-amide; $\quad 5 \beta$-cholanic- $3 \alpha, 7 \beta$-diol- $N$ (carboxymethl)-amide; $5 \beta$-cholanic- $3 \alpha, 7 \alpha$-diol- $N$-(carboxymethl)amide; $\quad 5 \beta$-cholanic- $3 \alpha, 12 \alpha$-diol- $N$-(carboxymethl)-amide; and $5 \beta$-cholanic- $3 \alpha$-ol- $N$-(carboxymethl)-amide.

\section{Organic acid analysis}

Organic acids in the caecal contents were measured using HPLC (Shimadzu Corporation) with crotonic acid (Wako Pure Chemical Industries, Ltd) as an internal standard according to the method of Hoshi et al. ${ }^{(27)}$. The caecal contents were homogenised and neutralised with sodium hydroxide to prevent the extraction of SCFA. Fat-soluble substances in the supernatant were removed by chloroform, and the aqueous phase was passed through a membrane filter (cellulose acetate, $0.20 \mu \mathrm{m}$ pore size; DISMIC-13cp; Toyo Roshi Kaisha, Ltd). The samples were analysed by HPLC (Shimadzu Corporation) equipped with a solvent delivery system (SCL-10 AVP; Shimadzu Corporation), a double ionexchange column (Shim-Pack SCR-102H, $8 \times 300$ mm; Shimadzu) and an electro-conductivity detector (CDD-6A; Shimadzu Corporation). The mobile phase was $5 \mathrm{~mm}$ of $p$-toluenesulphonic acid, and the detection solution was $5 \mathrm{~mm}$ of $p$-toluenesulphonic acid containing $100 \mu \mathrm{M}$ of EDTA and $20 \mathrm{~mm}$ of bis-tris.

\section{Enumeration of the intestinal bacteria}

Intestinal bacteria in the caecal contents were determined as described in the previous study ${ }^{(28)}$ with some modifications. The diluted solutions of caecal contents were inoculated onto plates with XM-G agar (Nissui Pharmaceutical Co., Ltd) for Escherichia coli and with standard method agar (Nissui Pharmaceutical Co., Ltd) for B. coagulans. Colony counts were made after cultivation at $37^{\circ} \mathrm{C}$ for $20 \mathrm{~h} \mathrm{(E.} \mathrm{coli)} \mathrm{and} 3 \mathrm{~d}$ (bifidobacteria and lactobacilli) in an anaerobic chamber and at $55^{\circ} \mathrm{C}$ for $2 \mathrm{~d}$ (B. coagulans). The $\mathrm{CFU}$ per gram of wet caecal contents were calculated.

\section{Intraperitoneal glucose tolerance test}

The intraperitoneal glucose tolerance (IPGTT) test was conducted according to the method of Higuchi et al. ${ }^{(29)}$. After fasting for $16 \mathrm{~h}$, blood samples were collected for the controls from the tail vein with $50 \mathrm{IU} / \mathrm{ml}$ heparin and $200 \mathrm{kIU} / \mathrm{ml}$ aprotinin at 7 weeks, and the glucose solution was injected $(1 \mathrm{~g} / \mathrm{kg}$ body weight) into the intraperitoneal cavity. Plasma samples were obtained from the tail vein with heparin and aprotinin at 15, 30, 60 and $120 \mathrm{~min}$ after glucose administration. The blood glucose levels were analysed using a glucose CII test Wako kit (Wako Pure Chemical Industries, Ltd).

\section{Statistics}

All the results are expressed as mean values with their standard errors. Statistical significance for comparisons between the basal group and each CA-fed group was determined using Dunnett's test. A two-way ANOVA was performed in the CA-fed groups for $B$. coagulans lilac-01 and soya pulp; probability $<0.05$ was considered statistically significant. JMP pro 12.0.1 (SAS institute Inc.) was used for the statistical analysis.

\section{Results}

Dietary supplementation of CA significantly promoted faecal BA excretion in various molecular species, such as DCA, 12oLCA, $3012 \alpha$ and HDCA (Fig. 1). In particular, faecal excretion of DCA, one of the toxic secondary BA originating from CA, increased significantly in the CA-fed rats as expected (Dunnett's test with basal, $P<0 \cdot 05)$. Moreover, DCA concentration was extremely higher in rats fed prebiotics in the CA-fed groups. An unexpected increase was found in faecal excretion of MCA and DCA by pre-ingestion. Interestingly, such an increase in secondary BA by soya pulp was almost abolished when the rats were fed the form of synbiotics with $B$. coagulans.

In addition, no significant difference was found in body weight gain and total food intake among the groups. In CA-fed groups, ingestion of soya pulp decreased liver weight (two-way ANOVA, $P=0.0145$ ) (Table 2). Liver weights tended to increase in CA-fed rats compared with basal diet-fed rats. No differences were observed for the other tissue weights, such as the kidney, whole caecum, epididymal adipose tissue and retroperitoneal adipose tissue. As a liver damage marker, we determined plasma AST (Fig. 2(a)) and ALT (Fig. 2(b)). There was no significant difference in AST levels among CA-fed rats, although ALT levels increased slightly in Syn-fed rats compared with basal diet-fed rats (Dunnett's test with basal, $P<0 \cdot 05$ ). Plasma AST and ALT also tended to increase in CA-fed rats compared with basal diet-fed rats. No significant difference was found in NEFA among the groups (Fig. 2(c)). Plasma TAG concentration was reduced in CA-fed rats, with the exception of Pro-fed rats, compared with the level in rats fed the basal diet (Dunnett's test with basal, $P<0.05$ ) (Fig. 2(d)). In addition, there was an interaction between the effect of prebiotics and probiotics on 


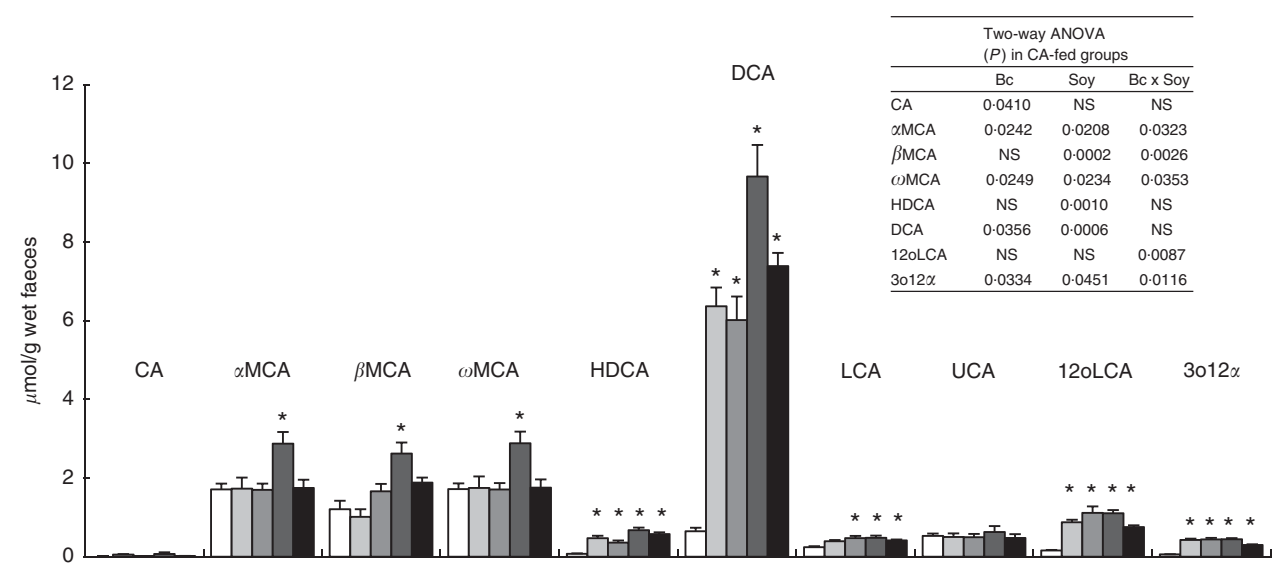

Fig. 1. Bile acid composition in wet faeces of rats fed basal (B) or cholic acid (CA)-supplemented diets in combination with Bacillus coagulans and soya pulp. Values are means $(n 5-7)$, with standard errors represented by vertical bars. * Significant difference from the value observed in rats fed the basal diet $(P<0.05$, Dunnett's test). $P$ values of the two-way ANOVA are shown in the inserted table for $B$. coagulans $(\mathrm{BC})$ and soya pulp (Soy). aMCA, $a$-muricholic acid; $\beta$ MCA, $\beta$-muricholic acid; $\omega \mathrm{MCA}$, $\omega$-muricholic acid; HDCA, hyodeoxycholic acid; DCA, deoxycholic acid; LCA, lithocholic acid; UCA, ursocholic acid; 12oLCA, 12-oxo-lithocholic acid; 3012a, $5 \beta$-cholanic acid-12a-ol-3-one; $\square, \mathrm{B} ; \mathrm{CA}: \square, \mathrm{Ct} ; \square$, probiotic diet; $\square$, prebiotic diet; $\square$, synbiotic diet.

Table 2. Growth parameters and tissue weights in rats fed experimental diets (Mean values with their standard errors)

\begin{tabular}{|c|c|c|c|c|c|c|c|c|c|c|c|c|c|}
\hline \multirow[b]{3}{*}{ Components } & & & \multicolumn{8}{|c|}{$\mathrm{CA}$} & & & \\
\hline & \multicolumn{2}{|l|}{ B } & \multicolumn{2}{|c|}{$\mathrm{Ct}$} & \multicolumn{2}{|c|}{ Pro } & \multicolumn{2}{|c|}{ Pre } & \multicolumn{2}{|c|}{ Syn } & \multicolumn{3}{|c|}{ Two-way ANOVA $(P)$ in CA-fed groups } \\
\hline & Mean & SEM & Mean & SEM & Mean & SEM & Mean & SEM & Mean & SEM & $\mathrm{Bc}$ & Soy & Bc $\times$ Soy \\
\hline \multicolumn{14}{|l|}{ Growth parameters } \\
\hline Initial BW (g) & 101 & 2 & 101 & 2 & 101 & 1 & 100 & 2 & 101 & 1 & NS & NS & NS \\
\hline Total food intake $(\mathrm{kg})$ & $15 \cdot 8$ & 0.2 & $15 \cdot 7$ & 0.4 & 16 & 0.4 & 16 & 0.6 & $15 \cdot 1$ & 0.3 & NS & NS & NS \\
\hline Final BW (g) & 343 & 7 & 333 & 4 & 344 & 8 & 327 & 8 & 336 & 6 & NS & NS & NS \\
\hline Tissue weight ( $\mathrm{g} / 100 \mathrm{~g}$ tissue) & & & & & & & & & & & NS & NS & NS \\
\hline Liver & 4.09 & 0.1 & 4.28 & 0.09 & $4 \cdot 25$ & 0.17 & 3.91 & 0.07 & 3.96 & $0 \cdot 13$ & NS & 0.0145 & NS \\
\hline Kidney & 0.61 & 0.01 & 0.64 & 0.01 & 0.64 & 0.01 & 0.62 & 0.01 & 0.62 & 0.01 & NS & NS & NS \\
\hline Whole caecum & 1.28 & 0.06 & $1 \cdot 31$ & 0.11 & $1 \cdot 2$ & 0.07 & 1.45 & 0.14 & 1.49 & 0.15 & NS & NS & NS \\
\hline Epididymal adipose tissue & 3.05 & 0.18 & $2 \cdot 69$ & 0.16 & 3 & 0.17 & 2.79 & 0.12 & $2 \cdot 79$ & 0.14 & NS & NS & NS \\
\hline Retroperitoneal adipose tissue & $2 \cdot 72$ & 0.11 & $2 \cdot 65$ & 0.11 & $2 \cdot 76$ & 0.15 & $2 \cdot 81$ & 0.09 & $2 \cdot 67$ & 0.13 & NS & NS & NS \\
\hline
\end{tabular}

CA, cholic acid; B, basal diet; Ct, control; Pro, probiotic diet; Pre, prebiotic; Syn, synbiotic diet; Bc, Bacillus coagulans; Soy, soya pulp; BW, body weight.

the plasma TAG concentration in CA-fed rats (two-way ANOVA, $P=0.0252)$. No significant difference was observed in plasma cholesterol concentrations (Fig. 2(e)). The ingestion of soya pulp increased plasma adiponectin concentrations in CA-fed rats (two-way ANOVA, $P=0.0023$ ) (Fig. 2(f)). The CA treatment promoted urinary $\mathrm{Cr}$ excretion as a marker of gut permeability (Dunnett's test with B, $P<0.05$ ) (Fig. 2(g)). Within CA-fed groups, gut permeability was alleviated by the ingestion of soya pulp (two-way ANOVA, $P=0.0036$ ). The ingestion of $B$. coagulans did not significantly influence gut permeability. There was no significant influence of the diets on plasma glucose concentration in the IPGTT study (Fig. 2(h)). Although there was no difference in liver TAG concentration among the groups (Fig. 2(i)), the ingestion of soya pulp significantly reduced liver cholesterol concentration in CA-fed rats (two-way ANOVA, $P=0 \cdot 0165$ ) (Fig. $2(\mathrm{j})$ ).

For caecal SCFA, there was a significant increase in the concentration of acetic acid in the Pre- and Syn-fed rats and in the concentration of succinic acid in Syn-fed rats compared with rats fed the basal diet (Dunnett's test, $P<0.05$ ) (Fig. 3(a)). There were subtle differences in the levels of other SCFA, but the levels were quite low in all cases. In CA-fed rats, ingestion of soya pulp increased acetic acid levels (two-way ANOVA, $P=0.0361$ ). In addition, a reduction of $\mathrm{pH}$ in caecal contents was found in relation to the increase in acetic acid concentrations (Fig. 3(b)). In caecal contents, B. coagulans was detected in Pro- and Syn-fed rats (Fig. 3(c)). The number of bifidobacteria and coliform was unaffected by the dietary intervention. A significant increase in the number of lactobacilli was observed following the ingestion of soya pulp in CA-fed rats (two-way ANOVA, $P=0 \cdot 0236$ ).

\section{Discussion}

MCA derived from CDCA are major molecular species of primary BA in rodents ${ }^{(30)}$; however, two major primary BA - CA and CDCA - are reported in humans ${ }^{(10)}$. Ageing increases DCA production in rodents and humans ${ }^{(20,21)}$. As ingesting a high-fat diet increases BA secretion ${ }^{(19,31)}$, CA supplementation in enterohepatic circulation is reasonable to mimic the BA 
(a)

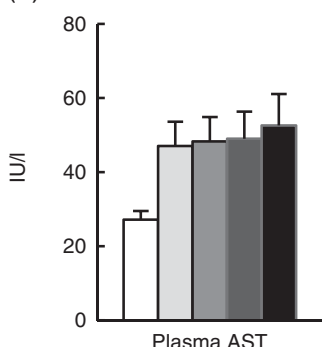

(f)

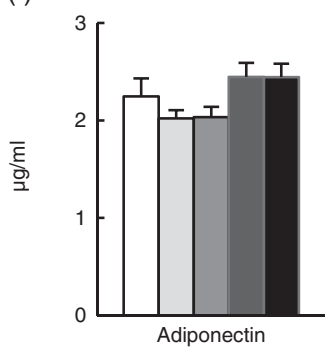

(i)

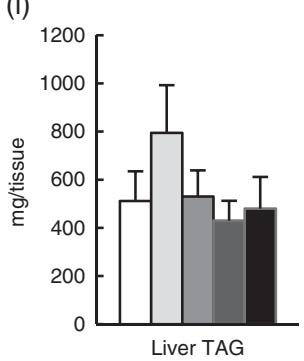

(b)

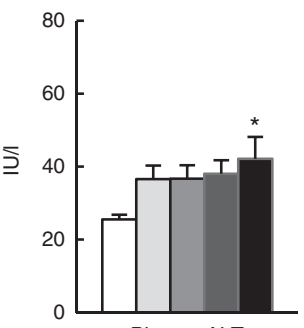

(g)

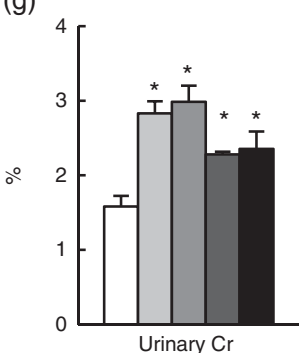

(j)

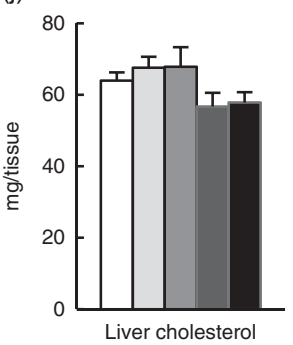

(c)

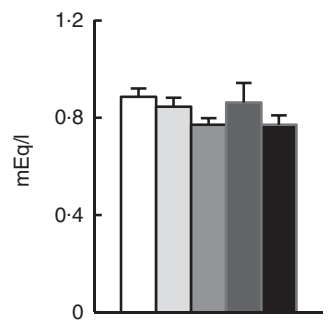

Plasma NEFA

(h)

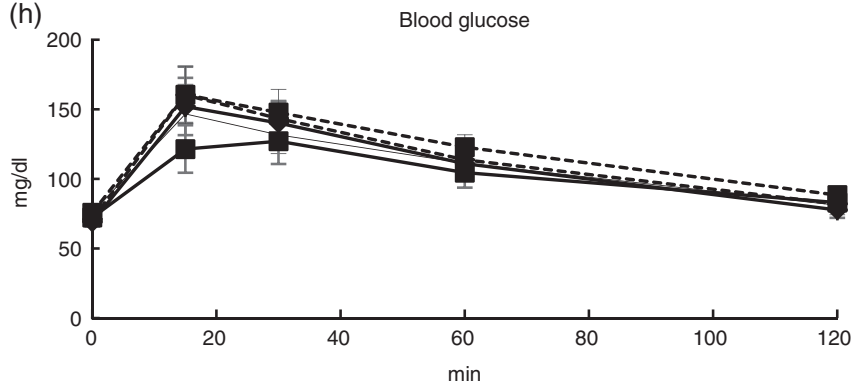

(e)
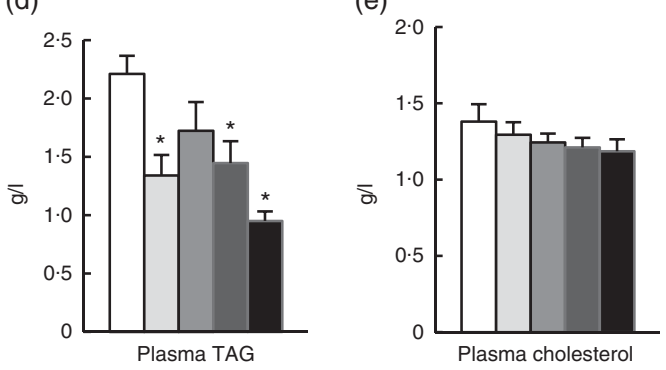

20

Fig. 2. Plasma parameters, urinary chromium excretion, intraperitoneal glucose tolerance test (IPGTT) and liver lipids of the rats fed basal (B) or cholic acid (CA)-supplemented diets in combination with Bacillus coagulans and soya pulp. Plasma concentrations of (a) aspartate aminotransferase (AST), (b) alanine aminotransaminase (ALT), (c) NEFA, (d) TAG, (e) cholesterol and (f) adiponectin. (g) Urinary chromium excretion as a marker for gut permeability at week 8. (h) Changes in plasma concentration during IPGTT at week 7. (i) Liver TAG accumulation, and (j) liver cholesterol accumulation at week 8. Values are means ( $n$ 5-7), with standard errors represented by vertical bars. ${ }^{*}$ Significant difference from the value observed in rats fed the basal diet $(P<0.05$, Dunnett's test). $P$ values of the two-way ANOVA are shown in the inserted table for $B$. coagulans (Bc)

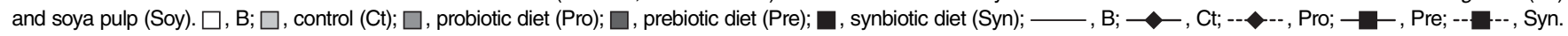

alteration in ageing and high-fat diet consumption. Generally, CA and taurocholic acid (TCA) are present at trace levels in the faeces ${ }^{(10,26)}$, but excess amounts of these primary or conjugated $\mathrm{BA}$ are found in rat faeces when the supplementation level of CA is $0.2 \%$ in the diet ${ }^{(32)}$. These observations suggest that CA supplementation to the diet beyond $0.2 \%$ is obviously an overdose for BA deconjugation and conversion ability of the intestinal bacteria. In contrast, such an excess increase in CA and TCA are not observed when rats are fed a CA diet at $0.05 \%$ or below ${ }^{(22)}$. Considering the gut BA metabolism, there must be an obstruction of BA deconjugation and hydroxylation in rats fed a CA supplementation $>0 \cdot 2 \%$.

There is a report showing that dietary CA supplementation at $0.5 \%$ promotes energy expenditure and abrogates diet-induced obesity in mice ${ }^{(33)}$. In our previous study ${ }^{(22)}$, we also observed a similar reduction in adipose tissue weight in rats fed a normalfat diet supplemented with $0 \cdot 2 \%$ of CA. However, such a reduction in adipose tissue weight disappeared in rats fed a diet that was supplemented with $0.05 \%$ of CA. In contrast, a microbiota analysis revealed an increase in Firmicutes in rats fed CA diets, regardless of the CA supplementation levels.
High-fat diet feeding is one of the practical models to reproduce diet-induced obesity and the metabolic syndrome. In preparation of a high-fat diet, we usually change the levels of two major ingredients at the same time, usually carbohydrates and lipids, which makes the interpretation of results more complicated because we need to consider the influence of two ingredients in the diet with regard to the results obtained. We can avoid such a complicated situation in case of the CA-supplementation study, because CA supplementation level at $0.5 \mathrm{~g} / \mathrm{kg}$ diet does not influence the composition of the major nutrients in the diets. We consider that the CA supplementation level in this experiment successfully mimics the BA metabolism found in ageing and in high-fat diet consumption without changing the composition of the major nutrients, as determined by the BA composition in the faeces.

First, we investigated BA metabolism in rats fed the CA diet. No difference in CA and TCA was confirmed among the groups, whereas a selective increase was found for $12 \alpha$-hydroxylated BA, such as DCA, 12oLCA and $3012 \alpha$, in rats fed the CA diet. This result indicates that the supplemented CA was completely converted to DCA via $7 \alpha$-hydroxylation in the large intestine, which represents the BA composition observed both in ageing 
(a)

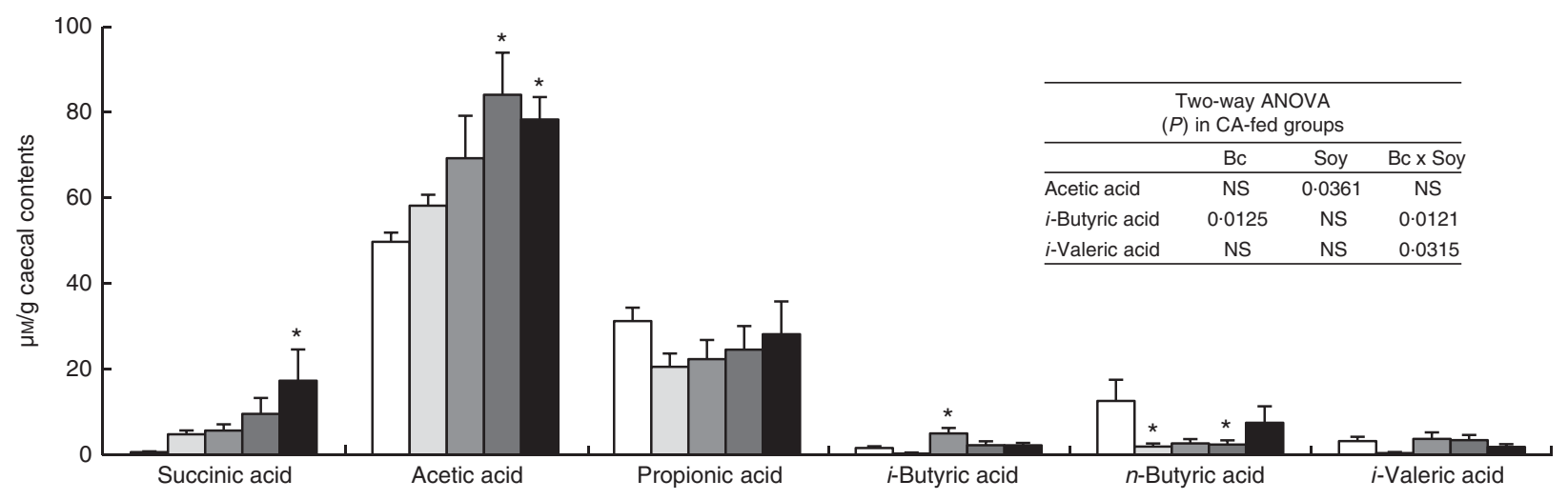

(b)

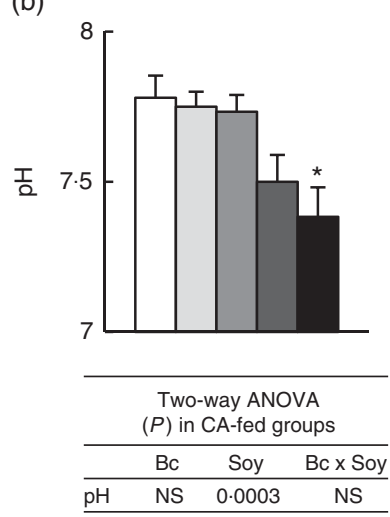

(c)

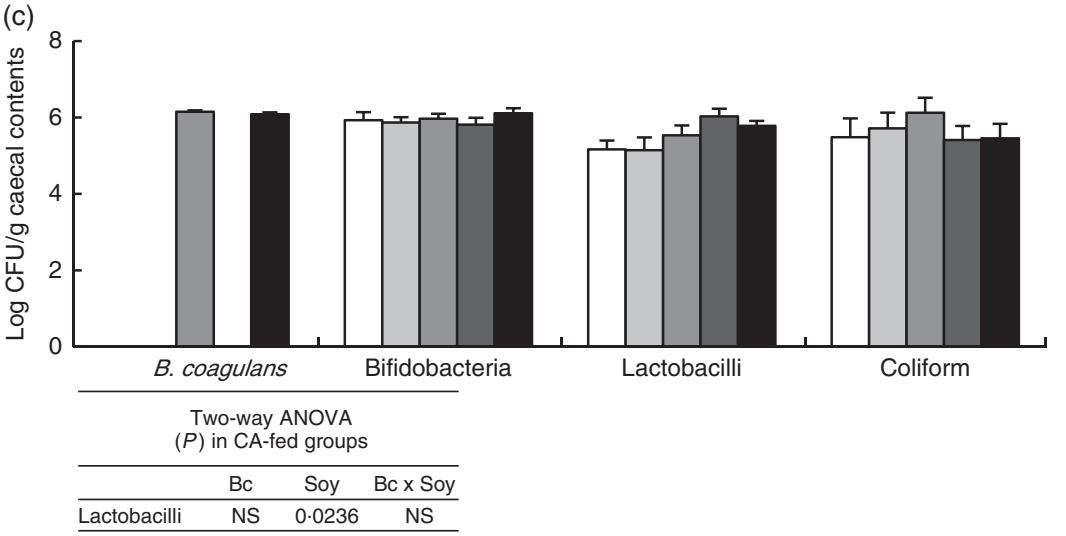

Fig. 3. Caecal parameters of rats fed basal (B) or the cholic acid (CA)-supplemented diets in combination with Bacillus coagulans and soya pulp at week 8. (a) Organic acid concentrations, (b) the $\mathrm{pH}$ of the caecal contents and (c) the number of bacteria in the caecal contents. Values are means ( $n$ 5-7), with standard errors represented by vertical bars. * Significant difference from the value observed in rats fed the basal diet $(P<0.05$, Dunnett's test). $P$ values of the two-way ANOVA are shown in the inserted table for B. coagulans (Bc) and soya pulp (Soy). CFU, colony forming unit; $\square, \mathrm{B} ; \mathrm{CA}: \square$, control (Ct); $\square$, probiotic diet; $\square$, prebiotic diet; 口, synbiotic diet.

and in high-fat diet consumption. By using CA supplementation, we evaluated whether B. coagulans and soya pulp influence BA metabolism. Interestingly, the ingestion of soya pulp increased DCA excretion, whereas the combination of soya pulp with $B$. coagulans normalised BA metabolism.

We observed a prominent increase in both MCA and DCA in rats fed Pro. In rodents, CA and MCA are usually secreted as taurine-conjugated forms in the bile juice. The taurine-conjugates are preferentially absorbed in the ileal epithelia, whereas the unconjugates are less absorbed $^{(12)}$. Many types of bifidobacteria and lactobacilli possess bile salt hydrolase (BSH) activity and are involved in BA deconjugation ${ }^{(12)}$. It is possible that these bacteria also increased in the intestinal contents of rats fed the Pre or Syn diet because soya pulp can be used as an energy source in the upper small intestine. The promotion of deconjugation and the inhibition of enterohepatic BA circulation by the increased population of endogenous lactic acid bacteria might be responsible for the increase in faecal DCA and MCA excretions. At an acidic $\mathrm{pH}$, achieved by luminal fermentation, unconjugated BA are protonated and precipitated, whereas taurine-conjugates remain solubilised ${ }^{(11)}$. Such unconjugated BA might be precipitated in the soya pulp and move into the large intestine, resulting in an increase in secondary BA. It is notable that excess increases in DCA and MCA were not observed in Syn-fed rats, regardless of soya pulp consumption. In general, BA deconjugation occurs gradually in the small intestine ${ }^{(34)}$, and almost no conjugated BA are found in the caecal contents ${ }^{(22)}$. However, in case of rats fed Syn, a massive amount of B. coagulans might exist in the small intestine due to the existence of soya pulp. It is possible that a reduction in BSH activity in the small intestine enables the suppression of DCA production if the B. coagulans does not display BSH activity.

Secondary BA are highly hydrophobic and can be absorbed in the large intestine, and thereby increase the risk for cancer development in the liver and large intestine. In a separate study $^{(35)}$, we observed that DCA is responsible for the proliferation and migration of vascular smooth muscle cells, indicating that an increased DCA concentration is involved in the development of various disorders. DCA is also shown to disrupt barrier function in gut epithelia in vivo ${ }^{(36)}$, and the penetration of exogenous substances is considered to induce unnecessary inflammatory responses in the liver and other gastrointestinal tissues. However, this study showed that soya pulp itself increased DCA production in the large intestine without increasing gut permeability. This result indicated that DCA itself might not necessarily be responsible for the CA-induced increase in gut permeability. In this study, we found that in rats fed the CA diet, ingesting soya pulp promoted 
barrier function. Acetate and butyrate suppressed colonic permeability in ex vivo and culture studies ${ }^{(37)}$. It is possible that the increase in acetic acid concentration in the luminal contents is involved in the normalisation of gut permeability by the ingestion of soya pulp. It could be advantageous to use a combination of soya pulp and $B$. coagulans to reduce the unnecessary increase in secondary BA production in the large intestine.

In the present study, the ingestion of soya pulp improved lipid metabolism under CA supplementation, although no positive effect was found on glucose tolerance. According to the manufacturer's instruction, the soya pulp used in the study contained $31 \mathrm{~g}$ of proteins, $8 \mathrm{~g}$ of carbohydrates, $17 \mathrm{~g}$ of lipids and $36 \mathrm{~g}$ of dietary fibre ${ }^{(6)} / 100 \mathrm{~g}$. The actual concentration of soya protein was approximately $1.5 \%$ in the diet. Soya pulp or soya protein is known to decrease liver lipid concentrations and lower the incidence of atheroscrelotic lesions ${ }^{(38)}$, but the supplementation levels of soya pulp and soya protein were quite low in this study. We have already reported a decrease in adiponectin concentration by CA ingestion ${ }^{(22)}$, but the mechanism is still unclear. However, such increases in plasma adiponectin concentrations by the ingestion of soya pulp under the consumption of the CA diet are considered to be protective against many aspects of the metabolic disorder ${ }^{(39)}$.

We usually consider that prebiotics are effective for health maintenance. In this study, some promotional effects of prebiotics were actually found in intestinal permeability and plasma adiponectin concentrations. However, this study also indicates that consumption of prebiotics was not necessarily 'almighty' in health maintenance. As a probiotic, B. coagulans might not be active in Pro-fed rats as judged by the $\mathrm{pH}$ in the caecal contents and DCA concentration in the faeces, indicating that they need an accessible form of energy source. We suggest that synbiotics will be an option to avoid such unfavourable situations. At this moment, there is no gold standard of a specific combination of prebiotics and probiotics. Careful management is required to find such suitable combinations.

In conclusion, soya pulp increased BA excretion induced by the CA diet, but the increased BA excretion was abolished when rats were fed soya pulp with $B$. coagulans. The ingestion of soya pulp basically improved gut permeability and plasma adiponectin, as well as normalisation of liver weight induced by ingestion of the CA diet. These results suggest that a suitable combination of probiotics and prebiotics reduces the risk for the development of various gastrointestinal disorders provoked via an increase in secondary BA.

\section{Acknowledgements}

This study was supported by the Regional Innovation Strategy Support Program of the MEXT (Ministry of Education, Culture, Sports, Science and Technology) from the Japanese Government.

Y. L. and S. I. analysed the data and wrote the article; S. I. and K. Minamida designed the study; Y. L., R. Y., K. K., G.-H. J., M. T., H. S. and T. N. carried out the research; K. Minamida and K. Miwa provided essential materials; K. Minamida and $\mathrm{H}$. $\mathrm{H}$. formulated the questions.

There are no conflicts of interest.

\section{References}

1. Gibson GR \& Roberfroid MB (1995) Dietary modulation of the humans colonic microbiota: introducing the concept of prebiotics. J Nutr 125, 1401-1412.

2. Patel R \& DuPont HL (2015) New approaches for bacteriotherapy: prebiotics, new-generation probiotics, and synbiotics. Clin Infect Dis 60, Suppl. 2, S108-S121.

3. Ishizuka S, Iwama A, Dinoto A, et al. (2009) Synbiotic promotion of epithelial proliferation by orally ingested encapsulated Bifidobacterium breve and raffinose in the small intestine of rats. Mol Nutr Food Res 53, S62-S67.

4. Ara K, Meguro S, Hase T, et al. (2002) Effect of spore-bearing lactic acid-forming bacteria (Bacillus coagulans SANK 70258) administration on the intestinal environment, defecation frequency, fecal characteristics and dermal characteristics in humans and rats. Microb Ecology Health Dis 14, 4-13.

5. Fan PH, Zang MT \& Xing J (2015) Oligosaccharides composition in eight food legumes species as detected by highresolution mass spectrometry. J Sci Food Agric 95, 2228-2236.

6. Minamida K, Nishimura M, Miwa K, et al. (2014) Effects of dietary fiber with Bacillus coagulans lilac-01 on bowel movement and fecal properties of healthy volunteers with a tendency for constipation. Biosci Biotechnol Biochem 79, 1-6.

7. Uchida A, Nomura Y \& Takeuchi N (1980) Effect of cholic acid, chenodeoxycholic acid, and their related bile acids on cholesterol, phospholipid, and bile acid levels in serum, liver, bile, and feces of rats. J Biochem $\mathbf{8 7}, 187-194$.

8. Redinger RN (2003) The coming of age of our understanding of the enterohepatic circulation of bile salts. Am J Surg 185, 168-172.

9. Shneider BL (2001) Intestinal bile acid transport: biology, physiology, and pathophysiology. J Pediatr Gastroenterol Nutr 32, 407-417.

10. Ridlon JM, Kang DJ \& Hylemon PB (2006) Bile salt biotransformations by human intestinal bacteria. J Lipid Res $\mathbf{4 7}$, 241-259.

11. Dashkevicz MP \& Feighner SD (1989) Development of a differential medium for bile salt hydrolase-active Lactobacillus spp. Appl Environ Microbiol 55, 11-16.

12. Liong MT \& Shah NP (2005) Bile salt deconjugation ability, bile salt hydrolase activity and cholesterol co-precipitation ability of lactobacilli strains. Int Dairy J 15, 391-398.

13. Pereira DIA, McCartney AL \& Gibson GR (2003) An in vitro study of the probiotic potential of a bile-salt-hydrolyzing Lactobacillus fermentum strain, and determination of its cholesterol-lowering properties. Appl Environ Microbiol 69, 4743-4752.

14. Kim GB, Yi SH \& Lee BH (2004) Purification and characterization of three different types of bile salt hydrolases from Bifidobacterium strains. J Diary Sci 87, 258-266.

15. Grill JP, Schneider F, Crociani J, et al. (1995) Purification and characterization of conjugated bile salt hydrolase from Bifidobacterium longum BB536. Appl Environ Microbiol 61, $2577-2582$

16. Knarreborg A, Engberg RM, Jensen SK, et al. (2002) Quantitative determination of bile salt hydrolase activity in bacteria isolated from the small intestine of chickens. Appl Environ Microbiol 68, 6425-6428.

17. Ferrari A \& Beretta L (1977) Activity on bile acids of a Clostridium bifermentans cell-free extract. FEBS Lett 75, 163-165.

18. Degirolamo C, Modica S, Palasciano G, et al. (2011) Bile acids and colon cancer: solving the puzzle with nuclear receptors. Trends Mol Med 17, 564-572.

19. Reddy BS (1981) Diet and excretion of bile acids. Cancer Res 41, 3766-3768. 
20. Uchida K, Chikai T, Takase H, et al. (1990) Age-related changes of bile acid metabolism in rats. Arch Gerontol Geriatr 10, $37-48$.

21. Salemans JM, Nagengast FM, Tangerman A, et al. (1993) Effect of ageing on postprandial conjugated and unconjugated serum bile acid levels in healthy subjects. Eur J Clin Invest 23, 192-198.

22. Islam KBMS, Fukiya S, Hagio M, et al. (2011) Bile acid is a host factor that regulates the composition of the cecal microbiota in rats. Gastroenterology 141, 1773-1781.

23. Reeves PG, Nielsen FH \& Fahey GC (1993) AIN-93 purified diets for laboratory rodents: final report of the American Institute of Nutrition ad hoc committee on the reformulation of the AIN-76A rodents diet. J Nutr 123, 1939-1951.

24. Suzuki T \& Hara H (2010) Dietary fat and bile juice, but not obesity, are responsible for increase in small intestinal permeability induced through the suppression of tight junction protein expression in LETO and OLETF rats. Nutr Metab (Lond) 7, 19.

25. Folch J, Lee M \& Solane-Stanley GH (1956) A simple method for the isolation and purification of total lipids from animal tissues. J Biol Chem 226, 497-509.

26. Hagio M, Matsumoto M, Fukushima M, et al. (2009) Improved analysis of bile acids in tissues and intestinal contents of rats using LC/ESI-MS. J Lipid Res 50, 173-180.

27. Hoshi S, Sakata T, Mikuni K, et al. (1994) Galactosylsucrose and xylosylfructoside alter digestive tract size and concentrations of cecal organic acids in rats fed diets containing cholesterol and cholic acid. J Nutr 124, 52-60.

28. Minamida K, Ohashi M, Hara H, et al. (2006) Effects of ingestion of difructose anhydride III (DFA III) and the DFA IIIassimilating bacterium Ruminococcus productus on rat intestine. Biosci Biotechnol Biochem 70, 332-339.

29. Higuchi N, Hira T, Yamada N, et al. (2013) Oral administration of corn zein hydrolysate stimulates GLP-1 and GIP secretion and improves glucose tolerance in male normal rats and Goto-Kakizaki rats. Endocrinology 154, 3089-3098.

30. Botham KM \& Boyd GS (1983) The metabolism of chenodeoxycholic acid to $\beta$-muricholic acid in rat liver. Eur $J$ Biochem 134, 191-196.

31. Stadler J, Stern S, Yeung KS, et al. (1988) Effect of high fat consumption on cell proliferation activity of colorectal mucoa and on soluble faecal bile acids. Gut 29, 1326-1331.

32. Hagio M, Shimizu H, Joe GH, et al. (2015) Diet supplementation with cholic acid promotes intestinal epithelial proliferation in rats exposed to $\gamma$-radiation. Toxicol Lett 232, 246-252.

33. Watanabe M, Houten SM, Mataki C, et al. (2006) Bile acids induce energy expenditure by promoting intracellular thyroid hormone activation. Nature 439, 484-489.

34. Tannock GW, Tangerman A, Schaik AV, et al. (1994) Deconjugation of bile acids by lactobacilli in the mouse small bowel. Appl Environ Microbiol 60, 3419-3420.

35. Shimizu H, Hagio M, Iwaya H, et al. (2014) Deoxycholic acid is involved in the proliferation and migration of vascular smooth muscle cells. J Nutr Sci Vitaminol (Tokyo) 60, 450-454.

36. Stenman LK, Holma R, Eggert A, et al. (2013) A novel mechanism for gut barrier dysfunction by dietary fat: epithelial dysruption by hydrophobic bile acids. Am J Physiol 304, G227-G234.

37. Suzuki T, Yoshida S \& Hara H (2008) Physiological concentrations of short-chain fatty acids immediately suppress colonic epithelial permeability. Br J Nutr 100, 297-305.

38. Lo GS, Evans KS, Philip RR, et al. (1987) Effect of soy fiber and soy protein on cholesterol metabolism and atheroscrelosis in rabbits. Atheroscrelosis 64, 47-54.

39. Brochu-Gaudreau K, Rehfeldt C, Blouin R, et al. (2010) Adiponectin action from head to toe. Endocrine 37, 11-32. 